\title{
A Phase II study of Amrubicin and Carboplatin for Previously Untreated Patients with Extensive-Disease Small Cell Lung Cancer
}

Takaya Ikeda, ${ }^{1}$ Minoru Fukuda, ${ }^{2}$ Yoichi Nakamura, ${ }^{3}$ Akitoshi Kinoshita, ${ }^{4}$ Hiroaki Senju, ${ }^{5}$ Hirofumi Nakano, ${ }^{6}$ Takeshi Kitazaki, ${ }^{2}$ Daiki Ogawara, ${ }^{3}$ Hirokazu Taniguchi, ${ }^{1}$ Kohei Motoshima, ${ }^{3}$ Hiroyuki Yamaguchi, ${ }^{7}$ Katsumi Nakatomi, ${ }^{3}$ Midori Shimada, ${ }^{2}$ Seiji Nagashima, ${ }^{3}$ Kazuhiro Tsukamoto, ${ }^{8}$ Shigeru Kohno ${ }^{3}$; Nagasaki Thoracic Oncology Group

${ }^{1}$ Department of Medicine, Sasebo General Hospital; Nagasaki; ${ }^{2}$ Clinical Oncology Center, Nagasaki University Hospital; Nagasaki; ${ }^{3}$ Second Department of Internal Medicine, Nagasaki University Hospital; Nagasaki; ${ }^{4}$ Department of Medicine, Nagasaki Prefecture Shimabara Hospital; Shimabara; ${ }^{5}$ Department of Medicine, National Hospital Organization Nagasaki Medical Center; Nagasaki; ${ }^{6}$ Department of Respiratory Medicine, National Ureshino Medical Center; Saga; ${ }^{7}$ Department of Cellular Signaling, Graduate School of Medicine, The University of Tokyo; Tokyo; ${ }^{8}$ Department of Clinical Pharmacy, Nagasaki University School of Pharmaceutical Science; Nagasaki; Japan.

Short title: Phase II of Amrubicin and Carboplatin in SCLC

Address correspondence to: Minoru Fukuda, MD, PhD

Clinical Oncology Center, Nagasaki University Hospital, 1-7-1 Sakamoto, Nagasaki, 852-8501, Japan 
Phone: +81-(95)-847-1511; Fax: +81-(95)-841-9613; E-mail: mifukuda258@nifty.com

Key Words: amrubicin, carboplatin, small cell lung cancer, phase II study 


\section{ABSTRACT}

Background: Amrubicin is active in the treatment of extensive-disease small cell lung cancer (ED-SCLC), and carboplatin is an analogue of cisplatin with less non-hematological toxicity.

Purpose: To determine the efficacy and toxicity of amrubicin and carboplatin combination chemotherapy for previously untreated patients with ED-SCLC.

Patients and methods: Eligibility criteria were chemotherapy-naïve ED-SCLC patients, performance status $0-1$, age $\leq 75$, and adequate hematological, hepatic and renal function. Based on the phase I study, the patients received amrubicin $35 \mathrm{mg} / \mathrm{m}^{2}$ i.v. infusion on days 1,2 and 3, and carboplatin AUC 5 i.v. infusion on day 1. Four cycles of chemotherapy were repeated every 3 weeks.

Results: Thirty-five patients were enrolled and 34 patients were eligible and assessable for response, toxicity and survival. Patients' characteristics were as follows: male/female $=26 / 8$; performance status $(\mathrm{PS})$ 0/1 $=4 / 30$; median age (range) $=64$ (41-75); stage IV = 34. Evaluation of responses were 6 complete response (CR), 21 partial response (PR), and 7 stable disease (SD) (response rate 79.4\%, 95\%CI 63.6-88.5\%). Grade 3 and 4 leukopenia, neutropenia, and thrombocytopenia occurred in $59 \%$, $82 \%$, and $26 \%$, respectively. There were no treatment-related deaths or pneumonitis. Three patients experienced hypotension as an amrubicin infusion reaction. The median progression-free survival time was 6.5 months. The median overall survival time and 1-, 2- and 3-year survival rates were 15.6 months, 63\%, 28\% and 7\%, respectively.

Conclusions: Amrubicin and carboplatin was effective and tolerable as chemotherapy for previously untreated patients with ED-SCLC. Further investigation of 
amrubicin and carboplatin is warranted. 


\section{INTRODUCTION}

Lung cancer is the leading cause of cancer deaths worldwide. It is also the Japanese leading cause of death, with 70,293 deaths (19.7\% of all cancer deaths) in 2011 (1). Of all lung cancer cases, approximately 20\% are small cell lung cancer (SCLC) (2). Although this cancer is initially highly responsive to chemotherapy, the vast majority of patients will ultimately relapse and die of recurrent disease within 2 years. Recently, combination chemotherapy with irinotecan and cisplatin for extensive-disease (ED) SCLC produced equal or more survival benefit than etoposide and cisplatin, the worldwide standard regimen since 1981 (3-5). The therapy provided 2-year survival rates of only $8 \%$ to $19.5 \%$, with a median survival time of 9.3 to 12.8 months. Clearly, new and more effective agents against SCLC are needed.

Amrubicin is a completely synthetic anthracycline derivative characterized by a 9-amino group and a simple sugar moiety. The chemical structure and acute toxicity of amrubicin are similar to those of doxorubicin $(6,7)$; however, it shows almost no cardiotoxicity $(8,9)$. Amrubicin alone and combination chemotherapy with cisplatin exhibited high response rates of $75.8 \%$ and $87.8 \%$ and median survival times of 11.7 and 13.6 months in previously untreated ED-SCLC, respectively $(10,11)$. Carboplatin is a platinum derivative with less renal toxicity that caused less nausea and vomiting than cisplatin (12), and has been combined with other newer agents in chemotherapy for SCLC (13). Pharmacodynamic studies have been performed to predict the clearance and administer the appropriate dose of carboplatin to individual patients (14-16). Accordingly, we conducted a phase I study with escalating doses of amrubicin under a fixed AUC 5 of carboplatin, and found that a dose of $35 \mathrm{mg} / \mathrm{m}^{2}$ amrubicin was recommended in this regimen (17). In the phase I study, this regimen is associated with 
an acceptable tolerability profile, a highly effective response rate of $73 \%$ and median survival time of 13.6 months.

Based on these results, we conducted a phase II study of amrubicin and carboplatin therapy for previously untreated ED-SCLC. The main objectives of the study were to determine the efficacy and safety of this regimen.

\section{PATIENTS AND METHODS}

The study protocol was reviewed and approved by the ethics committee of each institution, and written informed consent was obtained from all study participants. This study was an independent collaborative (unsponsored) group study. The clinical trial registration number is UMIN000001115.

\section{Patients and evaluation}

Eligibility criteria for patients in this study included the following: a histologically and/or cytologically confirmed diagnosis of ED-SCLC (19); no prior chemotherapy or radiotherapy; age $\leq 75$ years; Eastern Cooperative Oncology Group (ECOG) performance status (PS) $\leq 1$; life expectancy $>12$ weeks; adequate bone marrow function (leukocyte count $\geq 4,000 / \mu \mathrm{L}$, platelet count $\geq 10.0 \times 10^{4} / \mu \mathrm{L}$, and hemoglobin level $\geq 9.0 \mathrm{~g} / \mathrm{dL}$ ); serum bilirubin level $\leq 1.5 \mathrm{mg} / \mathrm{dL}$; ALT and AST levels $\leq 2$ times the normal upper limit; serum creatinine level $\leq$ the normal upper limit; and no medical problems severe enough to prevent compliance with the protocol.

\section{Treatment and dose escalation}


Based on our phase I study (17), patients received $35 \mathrm{mg} / \mathrm{m}^{2}$ amrubicin on days 1, 2 and 3, and carboplatin with a target AUC 5 mg.min/ml on day 1. Carboplatin was administered during a 60 -min intravenous infusion of $250 \mathrm{ml}$ of $5 \%$ dextrose. Amrubicin was diluted in $50 \mathrm{ml}$ normal saline and administered as an intravenous injection. Granulocyte colony-stimulating factor (G-CSF) was administrated when the neutrophil count became $<1,000 / \mu \mathrm{l}$ and was discontinued when the count recovered to $>5,000 / \mu l$. The next cycle commenced after leukocyte and platelet counts reached at

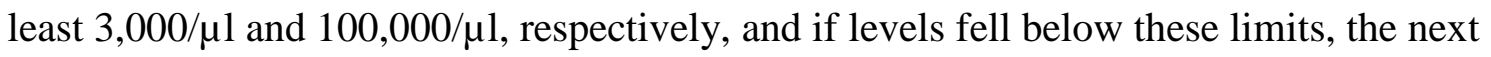
cycle was postponed until the counts recovered. Doses of amrubicin and carboplatin were reduced to $75 \%$ when grade 4 hematological toxicities occurred during the previous treatment cycle. In patients showing a response, this chemotherapy was repeated every 3 weeks for four cycles.

\section{Toxicity and response evaluation}

Eligibility, assessibility and tumor responses were determined by external reviewers. Drug toxicity was graded according to the NCI Common Terminology Criteria for Adverse Events v3.0 (CTCAE) (18). Before the first cycle, a blood cell count, urinalysis, and biochemistry tests were performed to assess renal and hepatic function and electrolytes. This monitoring was repeated during treatment, while other investigations were repeated, as necessary, to evaluate marker lesions. After the completion of treatment, each disease was assessed and tumors restaged. Tumor response was classified according to Response Evaluation Criteria in Solid Tumors (RECIST) and tumor markers were excluded from the criteria (19). Complete response (CR) was defined as the complete disappearance of all clinically detectable tumors for 
at least 4 weeks and no new lesions. Partial response (PR) was defined as at least a 30\% decrease in the sum of the longest diameters of the target lesion, taking as reference the baseline sum longest diameter, the required non-progression of non-target lesions and no new lesions. Stable disease (SD) included the regression of target lesions insufficient to meet the criteria for PR, a $<20 \%$ increase in the sum of the longest diameters of the target lesion, taking as reference the smallest sum longest diameters recorded since the treatment started, the required non-progression of non-target lesions and no new lesions. Progressive disease (PD) indicated $\mathrm{a} \geq 20 \%$ increase in the sum of the longest diameters of target lesion, taking as reference the smallest sum longest diameter recorded since the treatment started and/or unequivocal progression of existing non-target lesions and/or the appearance of new lesions. Evaluation of the objective tumor response for all patients was performed by external reviewers.

\section{Statistical analysis}

The primary endpoint of this study was the estimation of the objective response rate. The two-stage accrual design described by Simon was used (20). Assuming an overall response rate of $60 \%$ for standard therapy, a target response rate of $80 \%$ was established. Alpha $=0.05$ and beta $=0.20$, and the estimated required number of patients was 35. The upper limit of rejection was 25 responses (CR + PR). Overall survival was calculated by the Kaplan-Meier method (21).

\section{RESULTS}

Thirty-five patients from 7 institutions were enrolled in this trial between March 2008 and November 2011. All patients received the planned treatment and 34 
were evaluated for toxicity, response and survival. One patient was ineligible because of being judged to have limited disease by the external reviewers. The baseline patient characteristics are shown in Table 1.

\section{Treatment administration}

A total of 148 cycles of this therapy were administered with a median of 4 cycles per one patient: 1 cycle in 4 patients (12\%), 2 cycles in 2 (6\%), 3 in 1 (3\%), 4 in 11 (32\%), 5 in 3 (9\%), and 6 in 13 (38\%). In 4 patients who were administered only 1 cycle, 3 patients terminated the treatment because of hypotension. The remaining patient terminated the treatment because of a reduction of PS. Two terminated the treatment after 2 cycles because of PD and severe neutropenia. The latter was treated by 2 more cycles out of the study because the reduction dose was over the protocol. One patient achieved PR, but progressed and terminated the treatment after 3 cycles. The patients planned for 4 cycles, 16 patients were administered 5 or 6 cycles because continuing reduction of tumor size.

\section{Efficacy}

Of 35 patients, 34 were assessable for response. Objective tumor response was observed in 27 patients, with an overall response rate of 79.4\% [95\% confidence interval (CI) 63.6\% to 88.5\%] (Figure 1 and Table 2). Six (18\%) patients had CR. At the survival assessment in June 2013, six patients were still alive and the other 28 patients had died. The progression-free survival of 34 patients is shown in Figure 2A. Median progression-free survival time was 6.5 months. The overall survival of 34 
patients is shown in Figure 2B. Median survival time (MST) was 15.6 months and the 1-, 2-, 3-, 4-, and 5-year survival rates were $63 \%, 28 \%, 7 \%$, 7\%, and $0 \%$, respectively.

\section{Toxicity}

The worst grades of hematological and non-hematological toxicities experienced by each patient are listed Table 3. Of the 34 patients, 28 (82\%) experienced grade 3 or 4 hematological toxicity, and 21 (62\%) experienced grade 4 . The principal grade 3 or 4 toxicities were neutropenia and leukopenia in 28 (82\%) and 20 (59\%) patients, and the grade 4 toxicities were neutropenia, leukopenia and thrombocytopenia in 21 (62\%), 4 (12\%) and 4 (12\%), respectively. The common non-hematological adverse events was nausea in 18 patients (53\%). Hypotension was observed in 3 (9\%) patients after the first cycle of amrubicin on day 1 or 2 . There was no treatment-related death.

\section{DISCUSSION}

The present amrubicin combined with carboplatin therapy for previously untreated patients with ED-SCLC yielded 27 responders among 34 evaluable patients with an overall response rate of $79.4 \%$. Although this falls short of amrubicin/cisplatin (87.8\%) in a phase II trial (11), it was a high response compared with standard regimens of irinotecan/cisplatin (48-65\%) and etoposide/cisplatin (43.6\%-57\%) in phase III studies (3-5), and achieved the primary endpoint. Alternatively, the present MST of 15.6 months was longer than with the previous three regimens, which were 13.6, 9.3-12.8, 9.1-10.2 and 12.8 months, which were the MST of amrubicin/carboplatin for elderly (age $\geq 70$ ) patients with ED-SCLC (22). 
Recently, the Japanese Clinical Oncology Group (JCOG) reported a phase III trial of amrubicin/cisplatin for previously untreated ED-SCLC to compare combination therapy of irinotecan/cisplatin (23). Although the non-inferiority of amrubicin/cisplatin was not determined and overall survival was much worse than that of irinotecan/cisplatin, overall survival with the two regimens was excellent, 15.0 and 18.3 months, respectively. Amrubicin and irinotecan have shown activity in previously treated patients with ED-SCLC (24-26), and this might suggest that routine additional use of amrubicin with irinotecan, etoposide and platinum will prolong survival in ED-SCLC in Japan. In this JCOG study, dose of amrubicin was reduced $40 \mathrm{mg} / \mathrm{m}^{2}$ to 35 $\mathrm{mg} / \mathrm{m}^{2}$ because of severe myelotoxicities. The MSTs of amrubicin/cisplatin arm were 14.9 months $\left(40 \mathrm{mg} / \mathrm{m}^{2}\right)$ and 20.7 months $\left(35 \mathrm{mg} / \mathrm{m}^{2}\right)$, respectively. Considering with the activities of cisplatin and carboplatin and our amrubicin dose (35 mg/m²), our favorable MST of 15.6 months seems appropriate.

Based on our phase I trial (17), we used amrubicin $35 \mathrm{mg} / \mathrm{m}^{2}$ on days $1-3$ and carboplatin on day 1 with a target AUC of $5 \mathrm{mg} \cdot \mathrm{min} / \mathrm{ml}$ in the present study. Because the recommended dose (RD) of single-agent amrubicin is $45 \mathrm{mg} / \mathrm{m}^{2}(27)$, the $\mathrm{RD}$ of amrubicin is $10 \mathrm{mg} / \mathrm{m}^{2}$ lower in combination with carboplatin. In combination with cisplatin $60 \mathrm{mg} / \mathrm{m}^{2}$ on day 1 , the RD of amrubicin was initially $40 \mathrm{mg} / \mathrm{m}^{2}$ in a phase I-II trial (11). However, this level was too toxic and the dose of amrubicin was reduced to $35 \mathrm{mg} / \mathrm{m}^{2}$ during the phase III trial (23). The present phase II trial demonstrated the tolerability of $35 \mathrm{mg} / \mathrm{m}^{2}$ amrubicin; therefore, $35 \mathrm{mg} / \mathrm{m}^{2}$ was recommended for amrubicin combined with platinum.

The main severe toxicity associated with amrubicin is myelosuppression, with neutropenia seen more frequently than thrombocytopenia or anemia. In the present study, 
the incidence of grade 3 or 4 neutropenia was $82 \%$, which is lower than the incidence of $95.1 \%$ to $95.7 \%$ reported with amrubicin/cisplatin $(11,23)$. The rate of thrombocytopenia, which is associated with carboplatin toxicity, observed in this study (12\%) was also lower than the incidence of $24.4 \%$ to $27.1 \%$ reported with amrubicin/cisplatin $(11,23)$. This was perhaps due to the amrubicin dose difference, 35 $\mathrm{mg} / \mathrm{m}^{2}$ for our study and $40 \mathrm{mg} / \mathrm{m}^{2}$ in cisplatin studies. Because G-CSF was administered to patients with grade 3 or 4 neutropenia, it was commonly used. Unfortunately, one of our patients died due to severe hematological toxicities in the third cycle in the phase I trial (17), so we determined to decrease the dose from $80 \%$ to $75 \%$ in the next cycle in the phase II trial. Careful hematological toxicity control is essential in the therapeutic use of amrubicin. In most cases, myelosuppression was manageable with protocol-specific dose reductions, treatment delays, and G-CSF support, and there was no treatment-related death in the present study. The common non-hematological toxicity was nausea, and hypotension was observed in three patients (9\%). Although hypersensitivity reactions to carboplatin are well-known (28), the hypotension in the present study was observed immediately after administration of amrubicin and was considered to be an infusion reaction to amrubicin. This adverse effect had not been reported previously but was recently reported in a Japanese trial of amrubicin combined with S-1 (29). Pneumonitis, which is a difficult toxicity in cancer chemotherapy that has also been reported with amrubicin $(24,25)$, was not observed.

In conclusion, our phase II trial demonstrated the usefulness of amrubicin /carboplatin chemotherapy for previously untreated patients with ED-SCLC. This regimen is a convenient non-cisplatin treatment and is active in terms of the probability of response and survival compared with other regimens for SCLC. Further investigation 
of amrubicin/carboplatin is warranted.

\section{CONFLICT OF INTEREST STATEMENT}

None declared. 


\section{REFERENCES}

1. Vital Statistics of Japan (Ministry of Health, Labour \& Walfare).

2. Detterbeck FC (2000) Diagnosis and treatment of lung cancer, in An Evidence-Based Guide for the Practicing Physician. Philadelphia, PA, WB Saunders

3. Noda K, Nishiwaki Y, Kawahara M, Negoro S, Sugiura T, Yokoyama A, Fukuoka M, Mori K, Watanabe K, Tamura T, Yamamoto S, Saijo N (2002) Irinotecan plus cisplatin compared with etoposide plus cisplatin for extensive small-cell lung cancer. N Engl J Med 346:85-91

4. Hanna N, Bunn PA Jr, Langer C, Einhorn L, Guthrie T Jr, Beck T, Ansari R, Ellis P, Byrne M, Morrison M, Hariharan S, Wang B, Sandler A (2006) Randomized phase III trial comparing irinotecan/cisplatin with etoposide/cisplatin in patients with previously untreated extensive-stage disease small-cell lung cancer. J Clin Oncol 24:2038-2043

5. Lana PN Jr, Natale R, Crowley J, Lenz HJ, Redman MW, Carleton JE, Jett J, Langer CJ, Kuebler JP, Dakhil SR, Chansky K, Gandara DR (2009) Phase III trial of irinotecan/cisplatin compared with etoposide/cisplatin in extensive-stage small-cell lung cancer: clinical and pharmacogenomics results from SWOG S0124. J Clin Oncol 27:2530-2535

6. Ishizumi K, Ohashi N, Tanno N (1987) Stereospecific total synthesis of 9-aminoanthracyclines: (+)-9-amino-9-deoxydaunomycin and related compounds. J Org Chem 52:4477-85

7. Morisada S, Yanagi Y, Kashiwazaki Y, Fukui M (1989) Toxicological aspects of a novel 9-aminoanthracycline, SM-5887. Jpn J Cancer Res 80:77-82 
8. Suzuki T, Minamide S, Iwasaki T, Yamamoto H, Kanda H (1997) Cardiotoxicity of a new anthracycline derivative (SM-5887) following intravenous administration to rabbits: comparative study with doxorubicin. Invest New Drugs 15:219-225

9. Noda T, Watanabe T, Kohda A, Hosokawa S, Suzuki T (1998) Chronic effects of a novel synthetic anthracycline derivative (SM-5887) on normal heart and doxorubicin-induced cardiomyopathy in beagle dogs. Invest New Drugs 16:121-128

10. Yana T, Negoro S, Takada M, Yokota S, Takada Y, Sugiura T, Yamamoto H, Sawa T, Kawahara M, Katakami N, Ariyoshi Y, Fukuoka M (2007) Phase II study of amrubicin, in previously untreated patients with extensive-disease small cell lung cancer: West Japan Thoracic Oncology Group (WJTOG) study. Invest New Drugs 25:253-258

11. Ohe Y, Negoro S, Matsui K, Nakagawa K, Sugiura T, Takada Y, Nishiwaki Y, Yokota S, Kawahara M, Saijo N, Fukuoka M, Ariyoshi Y (2005) On behalf of the Amrubicin SCLC Study Group. Phase I-II study of amrubicin and cisplatin in previously untreated patients with extensive-stage small-cell lung cancer. Ann Oncol 16:430-436

12. Lokich J, Anderson N (1998) Carboplatin versus cisplatin in solid tumors: an analysis of the literature. Ann Oncol 9:13-21

13. Go RS, Adjei AA (1999) Review of the comparative pharmacology and clinical activity of cisplatin and carboplatin. J Clin Oncol 17:409-422

14. Egorin MJ, Van Echo DA, Olman EA, Whitacre MY, Forrest A, Aisner J (1985) Prospective validation of a pharmacologically based dosing schema for the cis-diamminedichloroplatinum (II) analogue diamminecyclobutanedicarboxylato-platinum. Cancer Res 45:6502-6506 
15. Calvert AH, Newell DR, Gumbrell LA, O’Reilly S, Burnell M, Boxall FE, Siddik ZH, Judson IR, Gore ME, Wiltshaw E (1989) Carboplatin dosage: prospective evaluation of a simple formula based on renal function. J Clin Oncol 7:1748-1756

16. Chatelut E, Canal P, Brunner V, Chevreau C, Pujol A, Boneu A, Roche H, Houin G, Bugat R (1995) Prediction of carboplatin clearance from standard morphological and biological patient characteristics. J Natl Cancer Inst 87:573-580

17. Fukuda M, Nakamura Y, Kasai T, Nagashima S, Nakatomi K, Doi S, Nakano H, Takatani H, Fukuda M, Kinoshita A, Soda H, Tsukamoto K, Oka M, Kohno S (2009) Phase I study of amrubicin and carboplatin for previously untreated patients with extensive-disease small cell lung cancer. J Thorac Oncol 4:741-745

18. Common Terminology Criteria for Adverse Events v3.0 (CTCAE), Bethesda, MD, National Cancer Institute (2006) Available at: http://ctep.cancer.gov/protocolDevelopment/electronic_applications/docs/ctcaev3.pd $\mathrm{f}$

19. Therasse P, Arbuck SG, Eisenhauer EA, Wanders J, Kapian RS, Rubinstein L, Verweij J, Van Giabbeke M, can Oosterom AT, Christian MC, Gwyther SG (2000) New guidelines to evaluate the response to treatment in solid tumors. J Natl Cancer Inst 92:205-216

20. Simon R. Optimal two-stage designs for phase II clinical trials (1989) Control Clin Trials 10:1-10

21. Kaplan EL, Meier P (1958) Nonparametric estimation from incomplete observations. J Am Stat Assoc 53:457-481

22. Inoue A, Ishimoto O, Fukumoto S, Usui K, Suzuki T, Yokouchi H, Maemondo M, Kanbe M, Ogura S, Harada T, Oizumi S, Harada M, Sugawara S, Fukuhara T, 
Nukiwa T (2010) A phase II study of amrubicin combined with carboplatin for elderly patients with small-cell lung cancer: North Japan Lung Cancer Study Group Trial 0405. Ann Oncol 21:800-803

23. Kotani Y, Satouchi M, Ando M, Nakagawa K, Yamamoto N, Ichinose Y, Ohe Y, Nishio M, Hida T, Takeda K, Kimura T, Minato K, Yokoyama A, Atagi S, Shibata T, Fukuda H, Tamura T, Saijo N (2012) A phase III study comparing amrubicin and cisplatin (AP) with irinotecan and cisplatin (IP) for the treatment of extended-stage small cell lung cancer (ED-SCLC): JCOG0509. Proc Am Soc Clin Oncol 31:\#7003.

24. Onoda S, Masuda N, Seto T, Eguchi K, Takiguchi Y, Isobe H, Okamoto H, Ogura T, Yokoyama A, Seki N, Asaka-Amano Y, Harada M, Tagawa A, Kunikane H, Yokoba M, Uematsu K, Kuriyama T, Kuroiwa Y, Watanabe K (2006) Phase II trial of amrubicin for treatment of refractory or relapsed small-cell lung cancer: Thoracic Oncology Research Group Study 0301. J Clin Oncol 24:5448-5453

25. Ettinger DS, Jotte R, Lorigan P, Gupta V, Garbo L, Alemany C, Conkling P, Spigel DR, Dudek AZ, Shah C, Salgia R, McNally R, Renschier MF, Oliver JW (2010) Phase II study of amrubicin as second-line therapy in patients with platinum-refractory small-cell lung cancer. J Clin Oncol 28:2598-2603

26. Masuda N, Fukuoka M, Kusunoki Y, Matsui K, Takifuji N, Kudoh S, Negoro S, Nishioka M, Nakagawa K, Takada M (1992) CPT-11: a new derivative of camptothecin for the treatment of refractory or relapsed small-cell lung cancer. J Clin Oncol 10:1225-1229

27. Sugiura T, Ariyoshi Y, Negoro S, Nakamura S, Ikegami H, Takada M, Yana T, Fukuoka M (2005) Phase I/II study of amrubicin, a novel 9-aminoanthracycline, in patients with advanced non-small-cell lung cancer. Invest New Drugs 23:331-337 
28. Weidmann B, Mulleneisen N, Bojko P, Niederle N (1994) Hypersensitivity reaction to carboplatin. Report of two patients, review of the literature, and discussion of diagnostic procedures and management. Cancer 73:2218-2222

29. Murakami S, Oshita F, Sugiura M, Kondo T, Saito H, Yamada K (2013) Phase I/II study of amrubicin in combination with S-1 as second-line chemotherapy for non-small-cell lung cancer without EGFR mutation. Cancer Chemother Pharmacol 71:701-711 


\section{FIGURE LEGENDS}

\section{Figure 1.}

Waterfall plots for the degree of tumor shrinkage. Most patients achieved tumor shrinkage. Complete and partial responses were observed 6 and 21 patients, respectively, and the response rate was $79.4 \%$.

\section{Figure 2.}

A) Progression-free survival curve, and B) overall survival curve of the 34 patients enrolled in the present study. 
Table 1. Patient characteristics

\begin{tabular}{ll}
\hline Characteristics & No. of patients \\
\hline Gender & \\
Male & 26 \\
Female & 8 \\
Age (years) & \\
Median & 64 \\
Range & $41-75$ \\
Performance status (ECOG) & \\
0 & 4 \\
1 & 30 \\
Stage & \\
IV & 34 \\
\hline
\end{tabular}

ECOG, Eastern Cooperative Oncology Group.

Table 2. Tumor response

\begin{tabular}{lrrrrr}
\hline $\mathrm{n}$ & $\mathrm{CR}$ & $\mathrm{PR}$ & $\mathrm{SD}$ & $\mathrm{PD}$ & $\begin{array}{c}\text { Response rate }(\%) \\
(95 \% \mathrm{CI})\end{array}$ \\
\hline 34 & 6 & 21 & 7 & 0 & $79.4(63.6-88.5)$ \\
\hline
\end{tabular}


Figure 1

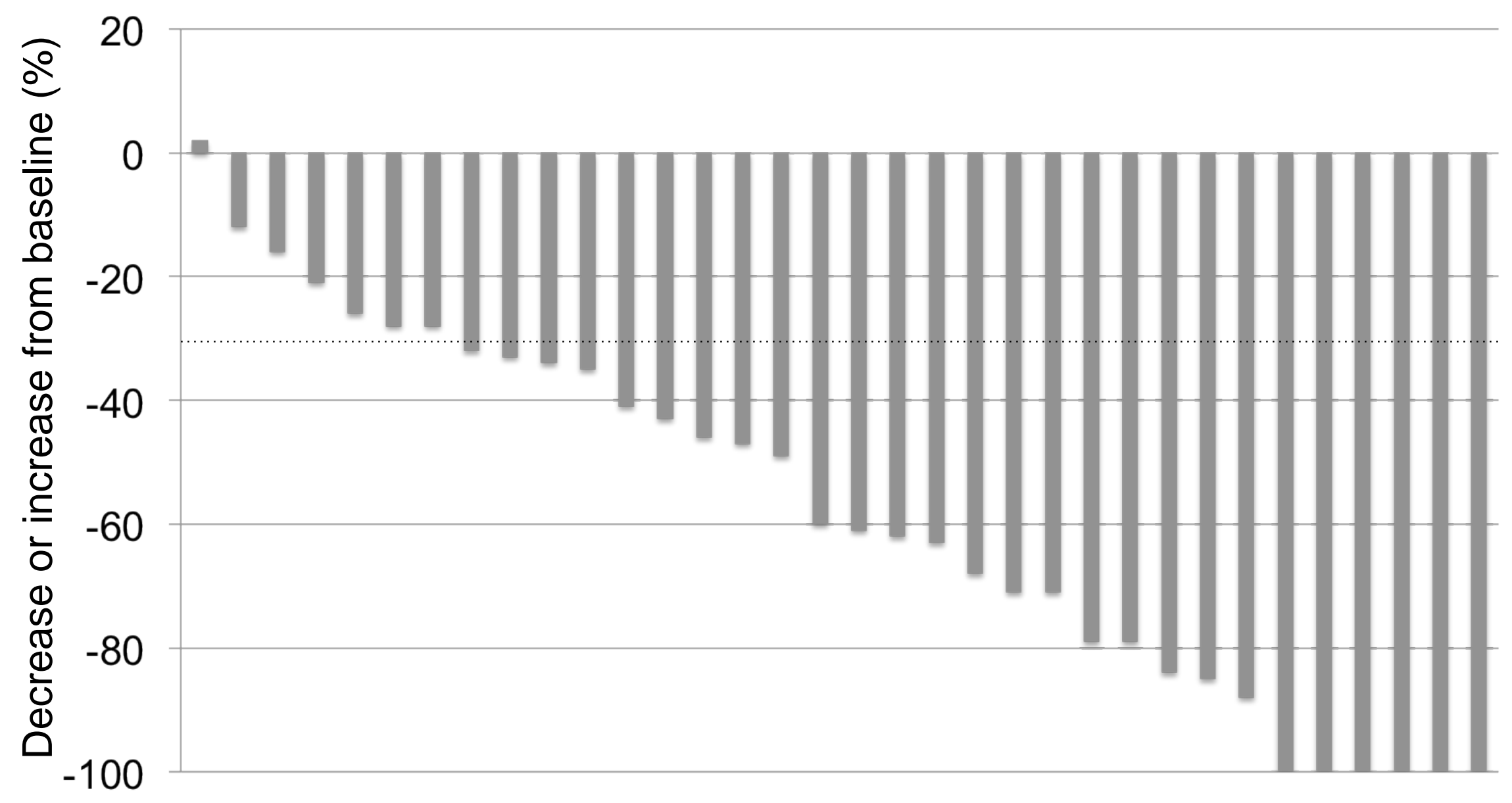


Figure 2A

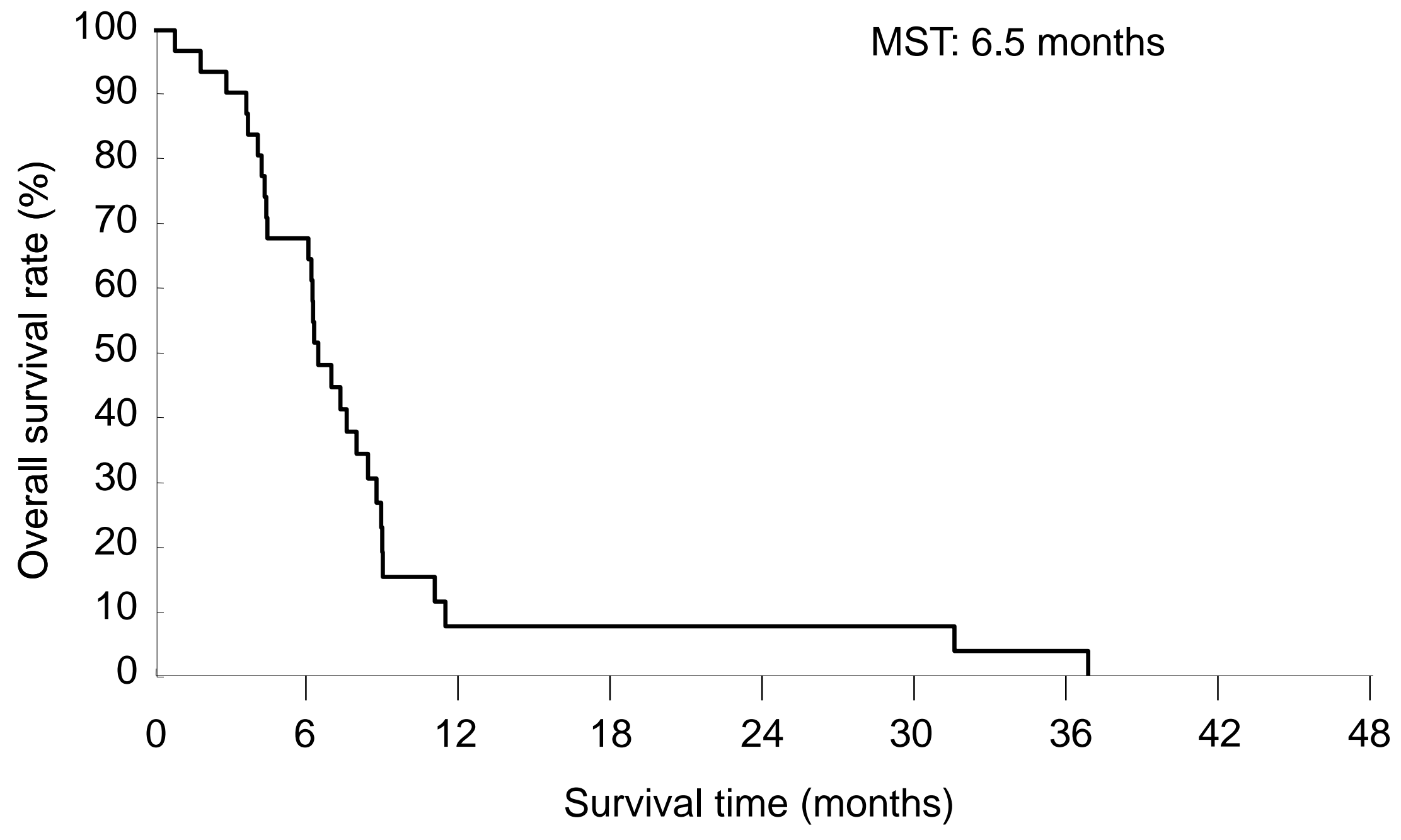


Figure 2B

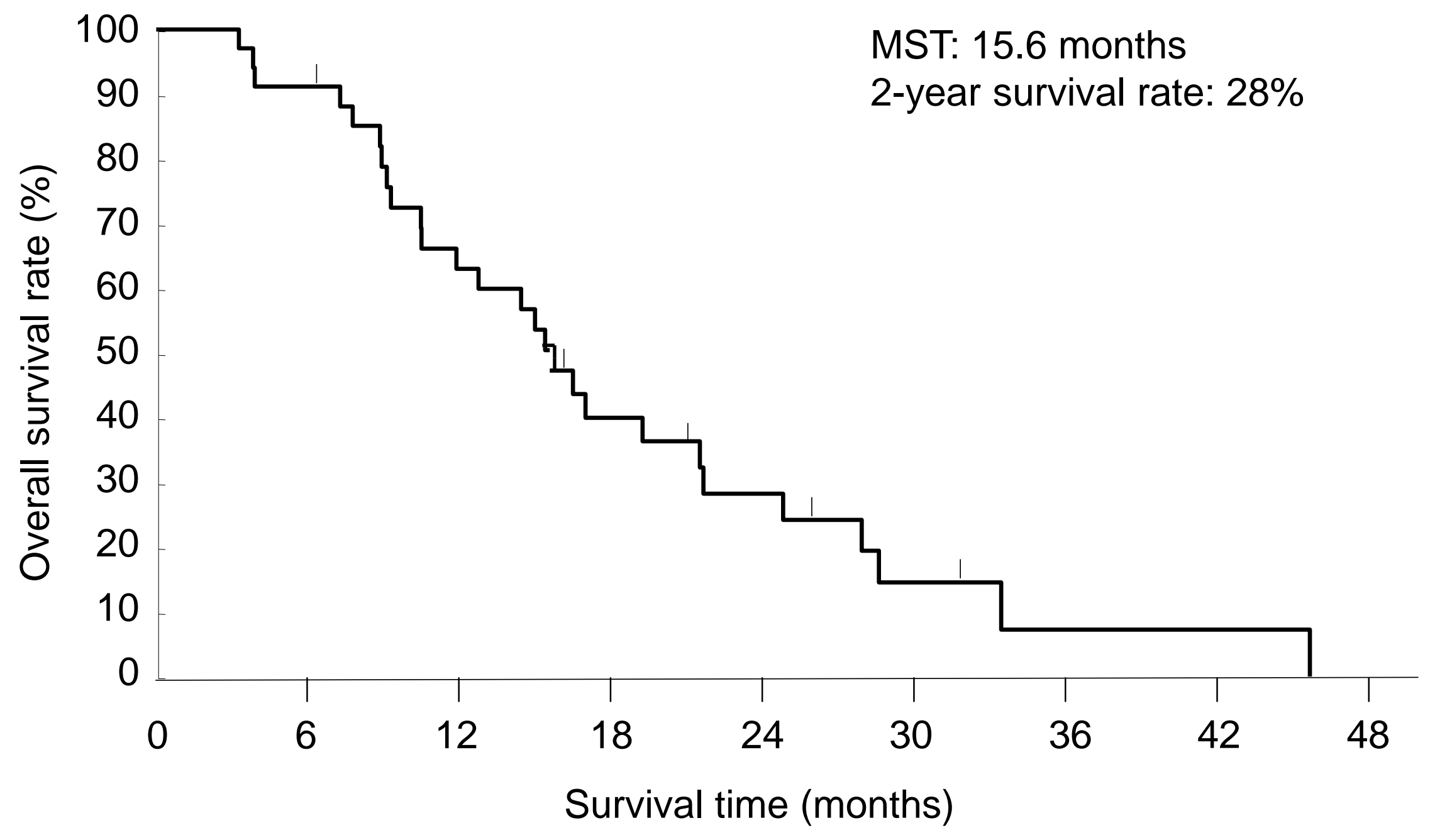

